\title{
ON THE LOW FREQUENCY ACOUSTICAL SCATTERING OF A PLANE WAVE FROM A SOFT SPINDLE AT NOSE-ON INCIDENCE*
}

\author{
BY ERGUN AR (University of Michigan)
}

1. Introduction. By means of a recently developed method it is now possible to solve iteratively the Dirichlet Problem [3], and the Neumann Problem [1] for the scalar Helmholtz equation $\left(\nabla^{2}+k^{2}\right) \phi=0$ in the regions exterior to a nonseparable body imbedded in the Euclidean 3-space; provided $k$, the complex wave number, is sufficiently small, and the solution of the Laplace's equation can be obtained for the body in question.

In what follows we shall consider one such body, namely, a spindle (a football shaped object).

We shall first obtain the potential Dirichlet Green's function for the spindle. Then by means of the above mentioned method we shall express, in the form of integrals, the scalar scattered field of a plane wave which is incident nose-on upon the spindle.

2. The Geometry of the Problem. The bispherical coordinate system $(\alpha, \beta, \phi)$, which is suitable for this problem, is given by

$$
\begin{gathered}
x=\frac{c \sinh \beta}{\cosh \beta-\cos \alpha}, \quad y=\frac{c \sin \alpha \sin \phi}{\cosh \beta-\cos \alpha}, \quad z=\frac{c \sin \alpha-\cos \phi}{\cosh \beta-\cos \alpha}, \\
h_{\beta}=h_{\alpha}=\frac{c}{\cosh \beta-\cos \alpha}, \quad h_{\phi}=\frac{c \sin \alpha}{\cosh \beta-\cos \alpha}, \quad c \text { is a positive real constant, }
\end{gathered}
$$

where

$$
h_{\alpha}^{2}=\left(\frac{\partial x}{\partial \alpha}\right)^{2}+\left(\frac{\partial y}{\partial \alpha}\right)^{2}+\left(\frac{\partial z}{\partial \alpha}\right)^{2}
$$

and similarly for $h_{\beta}$ and $h_{\phi}$.

In these coordinates

$$
\nabla^{2} \psi=\frac{1}{h_{\beta}^{3}}\left[\frac{\partial}{\partial \beta}\left(h_{\beta} \frac{\partial \psi}{\partial \beta}\right)+\frac{1}{\sin \alpha} \frac{\partial}{\partial \alpha}\left(h_{\beta} \sin \alpha \frac{\partial \psi}{\partial \alpha}\right)+\frac{h_{\beta}}{\sin ^{2} \alpha} \frac{\partial^{2} \psi}{\partial \phi^{2}}\right]
$$

The range of the variables are $-\infty<\beta<\infty, 0<\alpha<\pi, 0<\phi<2 \pi$.

The necessary and sufficient conditions for the separability of the Laplace (and Helmholtz) equations in various coordinate systems are given by Moon and Spencer [5]. Using their criteria for separation, we see that the Laplace equation is not simply separable in bispherical coordinates but is partially separable (whereas the Helmholtz equation is nonseparable in either sense).

In the bispherical coordinates the surface $\alpha=\alpha_{1}$ (const.) is a surface formed by rotating about the $x$-axis that part of the circle, in the $x-z$ plane, of a radius $c \operatorname{cosec} \alpha_{1}$

${ }^{*}$ Received February 7, 1966; revised manuscript received September 26, 1966. This work was supported by the National Science Foundation under grant GP-4581, and the Air Force Cambridge Research Laboratories under contract $A F-19(628)-4328$ 
with center $x=0, x=c \cot \alpha_{1}$. This surface of revolution is called a spindle. All the surfaces of constant $\alpha$ go through two points $x= \pm c(y=z=0)$; and at these points $\beta= \pm \infty$ respectively. The surface $\alpha=0$ is the $x$-axis for $x>c$ plus the sphere at infinity; the surface $\alpha=\pi / 2$ is the sphere of radius $c$ with center at the origin; and the surface $\alpha=\pi$ is the $x$-axis for $x<c$. The exterior region we are concerned with is $\alpha_{1} \geq$ $\alpha \geq 0, \infty>\beta>-\infty, 2 \pi \geq \phi \geq 0$.

3. The Potential Dirichlet Green's function for the spindle. The Jacobian of the transformation (2.1) is

$$
\frac{\partial(x, y, z)}{\partial(\alpha, \beta, \phi)}=\frac{c^{3} \sin \alpha}{(\cosh \beta-\cos \alpha)^{3}}
$$

Also

$$
\delta\left(x-x_{0}\right) \delta\left(y-y_{0}\right) \delta\left(z-z_{0}\right)=\left|\frac{\partial(x, y, z)}{\partial(\alpha, \beta, \phi)}\right|^{-1} \delta\left(\alpha-\alpha_{0}\right) \delta\left(\beta-\beta_{0}\right) \delta\left(\phi-\phi_{0}\right) .
$$

Therefore the potential Green's function satisfies the equation

$\nabla^{2} G_{0}\left(\alpha, \beta, \phi ; \alpha_{0}, \beta_{0}, \phi_{0}\right)=-4 \pi \frac{(\cosh \beta-\cos \alpha)^{3}}{c^{3} \sin \alpha} \cdot \delta\left(\alpha-\alpha_{0}\right) \delta\left(\beta-\beta_{0}\right) \delta\left(\phi-\phi_{0}\right)$

where $\left(\alpha_{0}, \beta_{0}, \phi_{0}\right)$ and $(\alpha, \beta, \phi)$ denote the source and the field points respectively, and $\nabla^{2}$ in bispherical coordinates is given by (2.2). The solution of Eq. (3.3) which vanishes on the boundary and satisfies the regularity condition $r G_{0}<\infty, r^{2}\left(\partial G_{0} / \partial r\right)<\infty$, as $r \rightarrow \infty$, can be found to be

$$
\begin{aligned}
& G_{0}\left(\xi, \beta, \phi ; \xi_{0}, \beta_{0}, \phi_{0}\right)=-\frac{1}{2 c} \sum_{-\infty}^{\infty} \exp \left[i m\left(\phi-\phi_{0}\right)\right](\cosh \beta-\xi)^{1 / 2}\left(\cosh \beta_{0}-\xi_{0}\right)^{1 / 2} \\
& \cdot \int_{\infty}^{\infty} d \nu \frac{1}{P_{i \nu-1 / 2}^{m}\left(\xi_{1}\right)} \frac{\exp \left[i \nu\left|\beta_{0}-\beta\right|\right]}{\sin (i \nu+m-1 / 2) \pi} \frac{\Gamma(i \nu+1 / 2-m)}{\Gamma(i \nu+1 / 2+m)} \\
& \begin{cases}{\left[P_{i \nu-1 / 2}^{m}(-\xi) P_{i \nu-1 / 2}^{m}\left(\xi_{1}\right)-P_{i \nu-1 / 2}^{m}(\xi) P_{i-1 / 2}^{m}\left(-\xi_{1}\right)\right] P_{i-1 / 2}^{m}\left(\xi_{0}\right),} & \xi \leq \xi_{0}, \\
P_{i \nu-1 / 2}^{m}(\xi)\left[P_{i \nu-1 / 2}^{m}\left(-\xi_{0}\right) P_{i \nu-1 / 2}^{m}\left(\xi_{1}\right)-P_{i \nu-1 / 2}^{m}\left(\xi_{0}\right) P_{i \nu-1 / 2}^{m}\left(-\xi_{1}\right)\right], & \xi \geq \xi_{0},\end{cases}
\end{aligned}
$$

where $\xi=\cos \alpha$.

With the substitution $s=i \nu-\frac{1}{2}$ the above representation for the Dirichlet Green's function is written as

$$
\begin{aligned}
& G_{0}=\frac{i}{2 c}(\cosh \beta-\cos \alpha)^{1 / 2}\left(\cosh \beta_{0}-\cos \alpha_{0}\right)^{1 / 2} \\
& \cdot \int_{-i \infty-1 / 2}^{i \infty-1 / 2} d s \frac{\exp \left[(s+1 / 2)\left|\beta-\beta_{0}\right|\right]}{\sin s \pi} \sum_{m=0}^{\infty} \epsilon_{m}(-1)^{m} \cos m\left(\phi-\phi_{0}\right) \frac{\Gamma(s+1-m)}{\Gamma(s+1+m)} \\
& \cdot\left\{P_{\cdot}^{m}(-\cos \alpha) P_{\cdot}^{m}\left(\cos \alpha_{0}\right)-\frac{P^{m}\left(-\cos \alpha_{1}\right)}{P_{s}^{m}\left(\cos \alpha_{1}\right)} P^{m} \cdot(\cos \alpha) P^{m}\left(\cos \alpha_{0}\right)\right\}, \quad \alpha_{1}>\alpha \geq \alpha_{0} .
\end{aligned}
$$

We now make the following observations in connection with the nature of the poles of the integrand:

${ }^{1}$ Equality is understood in the sense of distributions. We shall assume the usual $\delta$-function formalism such as substitution, integration by parts, etc., and for proofs refer to e.g., Gelfond and Schilow [2]. 
(1) First we note that since the behavior of $P_{b}^{m}(-\cos \alpha) P_{b}^{m}\left(\cos \alpha_{0}\right)$ is the same as $P_{b}^{m}\left(-\cos \alpha_{1}\right) / P_{b}^{m}\left(\cos \alpha_{1}\right) \cdot P_{b}^{m}(\cos \alpha) P_{s}^{m}\left(\cos \alpha_{0}\right)$ for large $|s|$. Therefore, we only need to study the integrand in the half plane $\operatorname{Re} s<-\frac{1}{2}$.

(2) $(1 / \sin s \pi)(\Gamma(s+1-m) /(\Gamma(s+1+m))$ has $m$ poles of order two at $s=-1,-2$, $\cdots,-m$ and simple poles at $s=-m-n, n=1,2,3, \cdots$, .

(3) In the $s$-plane $P_{0}^{m}(\cos \alpha)$ is an entire function. Zeros of $P_{s}^{m}$ in the $s$-plane are all real and distinct. ${ }^{2}$

(4) In the s-plane, the zeros of $P_{b}^{m}(\cos \alpha), P^{m}(\cos \beta)$ are different for $\alpha \neq \beta$; the zeros of $P_{i}^{m}(-\cos \alpha), P_{\cdot}^{m}(\cos \alpha)$ are different.

(5) Recall that in constructing the Green's function in the variable $\alpha$ over the range $\alpha_{1}>\alpha>\alpha_{0}$, we used the proper combination of two functions.

$$
\begin{aligned}
& \phi_{1}=P_{a}^{m}(-\xi) P_{a}^{m}\left(\xi_{1}\right)=-P^{m}(\xi) P^{m}\left(-\xi_{1}\right), \\
& \phi_{2}=P_{a}^{m}(\xi) .
\end{aligned}
$$

If $s$ is an integer, then since

$$
P^{m}(\xi)=(-1)^{m} P^{m}(-\xi), \quad P^{m}\left(\xi_{1}\right)=(-1)^{m} P^{m}\left(-\xi_{1}\right),
$$

the Green's function $G_{0} \equiv 0$ for all $\alpha$ in $\alpha_{1}>\alpha>0$. This means that the factor $(1 / \sin s \pi)(\Gamma(s+1-m) /(\Gamma(s+1+m)$ has in fact no residue contribution to the integral and the only contribution is from the simple poles of $1 / P^{m}\left(\cos \alpha_{1}\right)$ at the noninteger real values of $s$ in the region $\operatorname{Re} s<-\frac{1}{2}$.

(6) Let $s=s$, denote the noninteger real solutions of the equation $P^{m}\left(\cos \alpha_{1}\right)=0$ in the region $\operatorname{Re} s<-\frac{1}{2}$ of the $s$-plane.

We can now, by means of the observations (1) through (6), write down the final residue series for $G_{0}$

$$
\begin{gathered}
G_{0}=\frac{\pi}{c}(\cosh \beta-\cos \alpha)^{1 / 2}\left(\cosh \beta_{0}-\cos \alpha_{0}\right)^{1 / 2}\left\{\sum_{m=0}^{\infty} \epsilon_{m}(-1)^{m} \cos m\left(\phi-\phi_{0}\right)\right. \\
\cdot \sum_{i} \frac{\exp \left[\left(s_{i}+1 / 2\right)\left|\beta-\beta_{0}\right|\right]}{\sin s_{i} \pi} \frac{\Gamma\left(s_{i}+1-m\right)}{\Gamma\left(s_{i}+1+m\right)} \frac{P_{s_{i}}^{m}\left(-\cos \alpha_{1}\right)}{\frac{d}{d s}\left\{P_{i}\left(\cos \alpha_{1}\right)\right\}_{\ldots, s_{i}}} \\
\left.\cdot P_{t_{i}}^{m}(\cos \alpha) P_{{ }_{i}}^{m}\left(\cos \alpha_{0}\right)\right\} \quad \alpha_{1}>\alpha>\alpha_{0}
\end{gathered}
$$

4. Dirichlet Green's function for the Helmholtz operator. We are seeking the Green's function for the surface $B$ of the spindle $\alpha=\alpha_{1}$ satisfying

(a) $\left(\nabla^{2}+k^{2}\right) G_{k}\left(p, p_{0}\right)=-4 \pi \cdot \delta\left[R\left(p, p_{0}\right)\right], p, p_{0} \in V$,

(b) $G_{k}\left(p_{B}, p_{0}\right)=0$,

(c) the radiation condition, $\lim _{r \rightarrow \infty}\left|r\left(\partial G_{k} / \partial r-i k G_{k}\right)\right|=0$, uniformly in all directions, where $V$ denotes the volume exterior to the spindle suface $B, p(\alpha, \beta, \phi)$ the field point, $p_{0}\left(\alpha_{0}, \beta_{0}, \phi_{0}\right)$ the source point, $p_{B}\left(\alpha_{1}, \beta, \phi\right)$ a point on the surface of the spindle

${ }^{2} \mathrm{H}$. M. Macdonald showed [4] that for $\mu$ real and $\mu>0, P^{-\mu}(\cos \alpha)$ can have no complex zeros. He also showed that $P_{.}^{\mu}(\cos \alpha), \mu>0$, has an infinite number of distinct real zeros and, in addition, at most $2 k$ complex zeros, where $k$ is the greatest integer contained in $\mu$. In our case $m=0,1,2 \cdots$ and we can exclude the possibility of complex zeros as can be seen from the relation $P_{e}^{-m}(\cos \alpha)=$ $(\Gamma(s-m+1)) /(\Gamma(s+m+1))(-1)^{m} P_{s}^{m}(\cos \alpha)$ for integer $m$. 
$\alpha=\alpha_{1} \cdot R\left(p, p_{0}\right)$ is the distance between $p$ and $p_{0}$. We note that

$$
r=\left((\cosh \beta+\cos \alpha)^{1 / 2} /(\cosh \beta-\cos \alpha)\right)^{1 / 2},
$$

and $r \rightarrow \infty$ if and only if $\alpha \rightarrow 0, \beta \rightarrow 0$. With our choice of $\delta$-function and the radiation condition, the free space Green's function is $e^{i k R} / R$; and the composition of $G_{k}$ into singular and regular parts is given by

$$
G_{k}\left(p, p_{0}\right)=\frac{\exp \left[i k R\left(p, p_{0}\right)\right]}{R\left(p, p_{0}\right)}+U_{k}\left(p, p_{0}\right)
$$

where $U_{k}$ satisfies

$$
\left(\nabla^{2}+k^{2}\right) U_{k}=0
$$

and the radiation condition $4.1 \mathrm{c}$.

In our case the Helmholtz equation is reduced from the wave equation by assuming the harmonic time dependence $e^{i \omega t}$.

We now state the result [3] which will be used to generate the scattered field. If

$$
\begin{aligned}
& \text { (a) } w: \bar{V} \rightarrow E^{1}, \\
& \text { (b) } w \varepsilon C^{2}(V) \\
& \text { (c) }|r w|<\infty, \quad\left|r^{2} \frac{\partial w}{\partial r}\right|<\infty \text { as } r \rightarrow \infty,
\end{aligned}
$$

then $w(p)$ satisfies the integral equation

$$
w(p)=\int_{V} G_{0}\left(p, p^{\prime}\right)^{2} w\left(p^{\prime}\right) d v^{\prime}+\int_{B} w\left(p_{B}\right) \frac{\partial}{\partial n} G_{0}\left(p, p_{B}\right) d \sigma_{B}
$$

where the volume element is given by

$$
d v=d x d y d z=\frac{\partial(x, y, z)}{\partial(\alpha, \beta, \phi)} d \alpha d \beta d \phi=\frac{c^{3} \sin \alpha}{(\cosh \beta-\cos \alpha)^{3}} d \alpha d \beta d \phi,
$$

the surface element (for the surface $\alpha=\alpha_{1}$ ) by

$$
d \sigma=\frac{c^{2} \sin \alpha_{1}}{\left(\cosh \beta-\cos \alpha_{1}\right)^{2}} d \beta d \phi ;
$$

$\partial / \partial n$ is the normal derivative (in the direction out of $V$ ) and is given by

$$
\frac{\partial}{\partial n}=-\frac{1}{h_{\alpha}} \frac{\partial}{\partial \alpha}=-\frac{1}{c}(\cosh \beta-\cos \alpha) \frac{\partial}{\partial \alpha} \text {. }
$$

We want to represent the regular part $U_{k}$ of the Green's function using the above Theorem. $U_{k}$ is not regular at infinity. There is more than one way of making $U_{k}$ regular. Although, for example, the function $e^{-i k r} U_{k}$ is regular, in a particular problem like ours the choice must be made more judiciously to simplify the resulting equations and to enable us to carry out the integrations eventually arising in connection with the iteration. Thus, we define

$$
\tilde{U}=e^{-i k f(\alpha, \beta)} U_{k}
$$

and call $f$ the "eikonal". 
5. A suitable choice for the eikonal $f(\alpha, \beta)$. We have

$$
\tilde{U}=e^{-i k f} U_{k}
$$

and

$$
\left(\nabla^{2}+k^{2}\right) e^{i k f} \tilde{U}=0
$$

Therefore,

$$
\begin{aligned}
\nabla^{2}\left(e^{i k f} \tilde{U}\right) & +k^{2} e^{i k f} \tilde{U}=\nabla \circ\left(i k e^{i k f} \tilde{U} \nabla f+e^{i k f} \nabla \tilde{U}\right)+k^{2} e^{i k f} \tilde{U} \\
& =e^{i k f}[1-(\nabla f \circ \nabla f)] k^{2} \tilde{U}+e^{i k f} \nabla^{2} \tilde{U}+2 i k e^{i k f} \nabla \tilde{U} \circ \nabla f+i k e^{i k f} \tilde{U} \nabla^{2} f=0
\end{aligned}
$$

or

$$
\nabla^{2} \tilde{U}+2 i k \nabla \tilde{U} \circ \nabla f+i k \tilde{U} \nabla^{2} f+[1-(\nabla f \circ \nabla f)] k^{2} \tilde{U}=0 .
$$

We see from (5.3) that the first natural simplification is achieved by setting

$$
\nabla f \circ \nabla f=1 \text {. }
$$

This is the "eikonal equation" for $f$.

6. Solution of the equation $\nabla f \circ \nabla f=1$. In bispherical coordinates

$$
\nabla \equiv \frac{1}{c}(\cosh \beta-\cos \alpha)\left\{\hat{e}_{\alpha} \frac{\partial}{\partial \alpha}+\hat{e}_{\beta} \frac{\partial}{\partial \beta}+\frac{\hat{e}_{\phi}}{\sin \alpha} \frac{\partial}{\partial \phi}\right\}
$$

with (6.1), (5.4) becomes

$$
\left(\frac{\partial f}{\partial \alpha}\right)^{2}+\left(\frac{\partial f}{\partial \beta}\right)^{2}=\frac{c^{2}}{(\cosh \beta-\cos \alpha)^{2}}
$$

We note that

$$
\cosh \beta-\cos \alpha=2 \sin \left(\frac{\alpha+i \beta}{2}\right) \cdot \sin \left(\frac{\alpha-i \beta}{2}\right)=2 \sinh \left(\frac{\beta+i \alpha}{2}\right) \sinh \left(\frac{\beta-i \alpha}{2}\right) .
$$

Let

$$
z=\beta+i \alpha, \quad \bar{z}=\beta-i \alpha
$$

then

$$
\frac{\partial f}{\partial \alpha}=i \frac{\partial f}{\partial z}-i \frac{\partial f}{\partial \bar{z}}, \quad \frac{\partial f}{\partial \beta}=\frac{\partial f}{\partial z}+\frac{\partial f}{\partial \bar{z}}
$$

therefore

$$
\left(\frac{\partial f}{\partial \alpha}\right)^{2}+\left(\frac{\partial f}{\partial \beta}\right)^{2}=4 \frac{\partial f}{\partial z} \cdot \frac{\partial f}{\partial \bar{z}}
$$

The equation to be solved is

$$
4 \frac{\partial f}{\partial z} \cdot \frac{\partial f}{\partial \bar{z}}=\frac{c^{2}}{4 \sinh ^{2}(z / 2) \sinh ^{2}(z / 2)} .
$$

We want to find a solution to this equation which is regular at infinity. Assume $f=$ $g(z)+g(\bar{z})$, then

$$
2 \frac{\partial g(z)}{\partial z}=\frac{c}{2 \sinh ^{2}(z / 2)}=-c \frac{\partial}{\partial z}(\operatorname{coth}(z / 2))
$$


Hence,

$$
\begin{gathered}
g(z)=-(c / 2) \operatorname{coth}(z / 2) \\
f=\frac{c}{2}\left\{\operatorname{coth}\left(\frac{\beta+i \alpha}{2}\right)+\operatorname{coth}\left(\frac{\beta-i \alpha}{2}\right)\right\}, \quad c>0 .
\end{gathered}
$$

From $r=(\cosh \beta+\cos \alpha)^{1 / 2} /(\cosh \beta-\cos \alpha)^{1 / 2}$, and (6.3) it is immediately seen that

$$
f=0(r) \quad \text { as } r \rightarrow \infty \quad(\alpha \rightarrow 0, \beta \rightarrow 0) .
$$

Now, making use of the expansion [Wilcox (6)] for $U_{k}$, which is absolutely and uniformly convergent outside of a sphere completely containing the spindle, we have,

$$
r U_{k}=e^{i k r} \sum_{n=0}^{\infty} \frac{f_{n}}{r^{n}}
$$

and, with $\tilde{U}=e^{-i k f} U_{k}$,

$$
r \tilde{U}_{k}=e^{i k(r-f)} \sum_{n=0}^{\infty} \frac{f_{n}}{r^{n}}
$$

so that

$$
|r \tilde{U}|<\infty \text { as } r \rightarrow \infty
$$

Also

$$
r^{2} \frac{\partial \tilde{U}}{\partial r}=r^{2} \frac{\partial}{\partial r}\left\{e^{-i k f} U_{k}\right\}=r^{2} \frac{\partial}{\partial r}\left\{\frac{e^{i k(r-f)}}{r} \sum_{n=0}^{\infty} \frac{f_{n}}{r^{n}}\right\}
$$

therefore,

$$
\left|r^{2} \partial \tilde{U} / \partial r\right|<\infty \text { as } r \rightarrow \infty .
$$

From (6.5), (6.6) we see that

$$
\tilde{U}=e^{-i k f} U_{k}
$$

is regular at infinity.

We also note that $\nabla^{2} f=0$; therefore equation (5.3) yields

$$
\nabla^{2} \tilde{U}=-2 i k \nabla \tilde{U} \circ \nabla f \text {. }
$$

But

$$
\nabla \tilde{U}=\nabla\left(e^{-i k f} U_{k}\right)=-i k e^{-i k f} U_{k} \nabla f+e^{-i k f} \nabla U_{k}
$$

therefore,

$\nabla \tilde{U} \circ \nabla f=-i k e^{-i k f} U_{k}(\nabla f \circ \nabla f)+e^{-i k f} \nabla U_{k} \circ \nabla f=-i k e^{-i k f} U_{k}+e^{-i k f} \nabla U_{k} \circ \nabla f$.

So we have

$$
\nabla^{2} \tilde{U}=-2\left(k^{2} U_{k}+i k \nabla U_{k} \circ \nabla f\right) e^{-i k f} .
$$

$\tilde{U}$ satisfies the hypothesis of the above theorem; taking $w(p)=\tilde{U}$ in equation (4.3) we have

$$
\tilde{U}\left(p, p_{0}\right)=\int_{V} d v^{\prime} G_{0}\left(p, p^{\prime}\right) \nabla^{2} \tilde{U}\left(p^{\prime}, p_{0}\right)+\int_{B} d \sigma_{B} \tilde{U}\left(p_{B}, p_{0}\right) \frac{\partial}{\partial n} G_{0}\left(p, p_{B}\right)
$$

where $\nabla^{2} U$ is given by (6.7) or (6.8). Substituting (6.7) into (6.9), we finally obtain 


$$
\begin{aligned}
\tilde{U}\left(p, p_{0}\right)=-2 i k \int_{V} d v^{\prime} G_{0}\left(p, p^{\prime}\right) \nabla f \circ \nabla \tilde{U}\left(p^{\prime}, p_{0}\right) & \\
& +\int_{B} d \sigma_{B} \tilde{U}\left(p_{B}, p_{0}\right) \frac{\partial}{\partial n} G_{0}\left(p, p_{B}\right) .
\end{aligned}
$$

7. Scattering of a plane wave by the spindle. We write (6.10) in the operator form where

$$
\tilde{U}=K \circ \tilde{U}+U^{(0)}
$$

$$
K: \tilde{U} \rightarrow K \circ \tilde{U}=-2 i k \int_{v} d v G_{0}\left(p, p^{\prime}\right) \nabla f \circ \nabla \tilde{U}
$$

and

$$
U^{(0)}=\int_{B} d \sigma_{B} \tilde{U}\left(p_{B}, p_{0}\right) \frac{\partial}{\partial n} G_{0}\left(p, p_{B}\right)
$$

The iterates are given by

$$
U^{(N)}=\sum_{n=0}^{N} K^{n} \circ U^{(0)}
$$

or by

$$
\tilde{U}^{(N)}=K \circ U^{(N-1)}+U^{(0)}, \quad N \geq 1 .
$$

On the surface of the spindle $\alpha=\alpha_{1}$

$$
\tilde{U}\left(p_{B}, p_{0}\right)=e^{-i k s\left(\alpha_{1}, \beta\right)} U_{k}\left(p_{B}, p_{0}\right) ;
$$

also the sum of the scattered field $U_{k}$ and the incident field $U^{i n c}$ vanish on the surface; therefore (7.3) becomes

$$
U^{(0)}=-\int_{B} e^{-i k f\left(\alpha_{1}, \beta\right)} U^{i n c}\left(p_{B}\right) \frac{\partial}{\partial n} G_{0}\left(p, p_{B}\right) d \sigma
$$

We now take the incident field to be a plane wave propagating in the direction of the negative $x$-axis, thus,

$$
U^{i n c}=e^{-i k x(\alpha, \beta)}, \quad x(\alpha, \beta)=\frac{c \sinh \beta}{\cosh \beta-\cos \alpha},
$$

then we may assume $m=0$ in the expressions for the static Green's function

$$
\begin{aligned}
G_{0}= & \frac{\pi}{c}(\cosh \beta-\cos \alpha)^{1 / 2}\left(\cosh \beta_{0}-\cos \alpha_{0}\right)^{1 / 2} \\
& \cdot \sum_{i} \frac{\exp \left[\left(s_{i}+1 / 2\right)\left|\beta-\beta_{n}\right|\right]}{\sin s_{i} \pi} \frac{P_{e_{i}}\left(\cos \alpha_{1}\right)}{(d / d s)\left\{P_{d}\left(\cos \alpha_{1}\right)\right\}_{\ell_{-d_{i}}}} P_{\bullet_{i}}\left(\cos \alpha_{)} P_{\bullet_{i}}\left(\cos \alpha_{0}\right) .\right.
\end{aligned}
$$

Observing that $\partial / \partial n=-\left(1 / h_{\alpha}\right)(\partial / \partial \alpha)=-(1 / c)(\cosh \beta-\cos \alpha)(\partial / \partial \alpha)$ and that $P_{. j}\left(\cos \alpha_{1}\right)=0$, from (7.8) we obtain

$$
\begin{aligned}
& \left.\frac{\partial G_{0}}{\partial n}\right|_{\alpha=\alpha_{2}}=\frac{\pi}{c^{2}} \sin \alpha_{1}\left(\cosh \beta-\cos \alpha_{1}\right)^{3 / 2}\left(\cosh \beta_{0}-\cos \alpha_{0}\right)^{1 / 2} \\
& \cdot \sum_{j} \frac{\exp \left(\left(s_{j}+1 / 2\right)\left|\beta-\beta_{n}\right|\right)}{\sin s_{j} \pi} \frac{P_{s_{j}}\left(-\cos \alpha_{1}\right)}{(d / d s)\left\{P_{s}\left(\cos \alpha_{1}\right)\right\}_{s=s_{i}}}\left\{\frac{d}{d \alpha}\left[P_{s_{j}}(\cos \alpha)\right]\right\}_{\alpha=\alpha_{1}} \cdot P_{d_{i}}\left(\cos \alpha_{0}\right) .
\end{aligned}
$$


Substituting (7.7) and (7.8) into (7.9) and noting that

$$
\int_{B} d \sigma_{B}=\int_{0}^{2 \pi} d \phi \int_{-\infty}^{\infty} \frac{c^{2} \sin \alpha_{1}}{\left(\cosh \beta-\cos \alpha_{1}\right)^{2}} d \beta,
$$

we obtain

$$
\begin{aligned}
U^{(0)}\left(\alpha_{0}, \beta_{1}\right)= & -2 \pi^{2} \sin ^{2} \alpha_{1}\left(\cosh \beta_{0}-\cos \alpha_{0}\right)^{1 / 2} \\
& \cdot \sum_{i} \frac{1}{\sin s_{j} \pi} \frac{P_{s_{i}}\left(-\cos \alpha_{1}\right)}{(d / d s)\left\{P_{s}\left(\cos \alpha_{1}\right)\right\}_{s-s_{j}}} \frac{d}{d \alpha}\left\{P_{s_{i}}(\cos \alpha)\right\}_{\alpha=\alpha_{s}} P_{s_{i}}\left(\cos \alpha_{0}\right) \\
& \cdot \int_{-\infty}^{\infty} d \beta \frac{\exp \left[-i k\left[f\left(\alpha_{1}, \beta\right)+x\left(\alpha_{1}, \beta\right)\right]\right] \exp \left[\left(s_{i}+\frac{1}{2}\right)\left|\beta-\beta_{0}\right|\right]}{\left(\cosh \beta-\cos \alpha_{1}\right)^{3 / 2}},
\end{aligned}
$$

where

$$
\begin{aligned}
& f\left(\alpha_{1}, \beta\right)=\frac{c}{2}\left\{\operatorname{coth}\left(\frac{\beta+i \alpha_{1}}{2}\right)+\operatorname{coth}\left(\frac{\beta-i \alpha_{1}}{2}\right)\right\}, \\
& x\left(\alpha_{1}, \beta\right)=\frac{c \sinh \alpha}{\cosh \beta-\cos \alpha_{1}},
\end{aligned}
$$

and we recall that $s_{j}$ are the real zeros of $P_{{ }_{i}}\left(\cos \alpha_{1}\right),\left(s_{i}<-\frac{1}{2}\right)$.

The iterates $U^{(N)}$ for $N \geqq 1$ are given by

$$
U^{(N)}=-2 i k \int_{V} d v^{\prime} G_{0}\left(p, p^{\prime}\right) \nabla f\left(\alpha^{\prime}, \beta^{\prime}\right) \circ \nabla U^{(N-1)}\left(\alpha^{\prime}, \beta^{\prime}\right)+U^{(0)}
$$

where

$$
\int_{V} d v^{\prime}=\int_{0}^{2 \pi} d \phi^{\prime} \int_{0}^{\pi} d \alpha^{\prime} \int_{-\infty}^{\infty} d \beta^{\prime} \frac{c^{3} \sin \alpha^{\prime}}{\left(\cosh \beta^{\prime}-\cos \alpha^{\prime}\right)^{3}} .
$$

Acknowledgment. The author is indebted to R. E. Kleinman for suggesting the problem.

\section{REFERENCES}

[1] Ergun Ar and R. E. Kleinman, The exterior Neumann problem for the 3-dimensional Helmholtz equation, Arch. Rational Mech. Anal. 23, 218-236 (1966)

[2] I. M. Gel'fand and G. E. Schilow, Verallgemeinerte Funktionen (distributionen). I, Veb Deutscher Verlag Der Wissenshaften, Berlin, 1962. (Translation from the Russian)

[3] R. E. Kleinman, The Dirichlet problem for the Helmholtz equation, Arch. Rational Mech. Anal. 18, 205229 (1965)

[4] H. M. Macdonald, Zeros of the spherical harmonic $P_{n}^{m}(\mu)$ considered as a function of $n$, Proc. London Math. Soc. (1), 31, 265 (1900)

[5] P. Moon and D. E. Spencer, Separability conditions for the Laplace and Helmholtz equations, J. Franklin Inst. 253, 585 (1952)

[6] C. H. Wilcox, A generalization of theorems of Rellich and Atkinson, Proc. Amer. Math. Soc. 7, 271276 (1956) 